\title{
Tunneling of condensate magnetization in a double-well potential
}

\author{
Ö. E. Müstecaplıoğlu, ${ }^{1}$ M. Zhang, ${ }^{2}$ and L. You ${ }^{3}$ \\ ${ }^{1}$ Koç University, Department of Physics, Rumelifeneri Yolu, 34450 Sariyer, Istanbul, Turkey \\ ${ }^{2}$ Center for Advanced Study, Tsinghua University, Beijing, 100084, China \\ ${ }^{3}$ School of Physics, Georgia Institute of Technology, Atlanta, Georgia 30332, USA
}

(Received 27 January 2005; published 31 May 2005)

\begin{abstract}
We study quantum dynamical properties of a spin-1 atomic Bose-Einstein condensate in a double-well potential. Adopting a mean field theory and single spatial mode approximation, we characterize our model system as two coupled spins. For certain initial states, we find full magnetization oscillations between wells not accompanied by mass (or atom numbers) exchange. We identify dynamic regimes of collective spin variables arising from nonlinear self-interactions that are different from the usual Josephson oscillations. We also discuss magnetization beats and incomplete oscillations of collective spin variables other than the magnetization. Our study points to an alternative approach to observe coherent tunnelling of a condensate through a (spatial) potential barrier.
\end{abstract}

DOI: 10.1103/PhysRevA.71.053616

PACS number(s): 03.75.Lm, 73.43.Jn, 75.40.Gb, 75.45.+j

In different branches of physics, various systems larger than atomic size have been examined to have signatures of purely quantum effects particularly in quantum coherence and tunnelling [1-3]. The macroscopic quantum tunnelling of magnetization (MQTM) [4-6] is such an effect that has been vigorously sought after and has been claimed to be observed in molecular nanomagnets of $\mathrm{Mn}_{12}$-acetate and $\mathrm{Fe}_{8}$ compounds $[7,8]$. The surprising event of MQTM was also believed to occur in iron storage protein ferritin [9]. Apart from its fundamental significance at the interface of quantum and classical realms, MQTM in a large spin system is expected to play a key role in realizing technological advances for future's quantum computers and for the newly emerging field of spintronics. Being macroscopic quantum objects themselves, optically trapped atomic Bose-Einstein condensates (BECs) $[10,11]$ are also proposed to be promising candidates for MQTM [12], though strictly speaking, one may classify such systems as mesoscopic as well.

In optical traps hyperfine spin degrees of freedom become accessible and for a condensate of atoms in the $F=1$ hyperfine ground state, its order parameter is a three component $\left(M_{F}=-1,0,+1\right)$ spinor. The tunnelling associated with the internal (spin) degree of freedom can be induced by a transverse magnetic field when it exceeds a critical value depending on the strength of magnetic spin dipole-dipole interaction [12]. Such Josephson oscillations with internal degrees of freedom can be compared to the well-known superfluid ${ }^{3} \mathrm{He}$ Josephson oscillations observed in recent years [13]. The tunnelling associated with the external (spatial) center of mass degree of freedom has been demonstrated with cold atoms [14-16] as well as atomic condensates in an optical lattice [17]. The underlying strong correlations in spin and motional degrees of freedom in such systems may eventually lead to novel applications in spintronics. Beyond the semiclassical macroscopic Josephson oscillation physics, two tunnelling coupled condensates can also display many particle quantum correlations when the strength of tunnelling coupling is tuned relative to the typical mean field interaction strength inside each well [18]. While this phenomenon is well understood for scalar condensates, it is so far unexplored for spin-1 condensates [19-21]. In this article, we propose a different and more direct mechanism for observing coherent condensate Josephson dynamics based on magnetization oscillations due to an external (spatial) Josephson coupling of a spin- 1 condensate in a double well potential, building upon previous studies of a spin $1 / 2$ condensate in a double well potential [22-24].

Our model system consists of a spin- 1 condensate in a double-well potential. When tunnelling through the potential barrier is weak, the wave function effectively behaves as a superposition of those localized in the left (L) and right (R) wells. In the mean field approximation, we write the three component order parameter as $\psi_{i}(\vec{r}, t)=\phi_{L i}(\vec{r}) \xi_{i}(t)$ $+\phi_{R i}(\vec{r}) \eta_{i}(t)$ with $i=-, 0,+$ respectively for each Zeeman state $\left|M_{F}\right\rangle . \phi_{\nu i}$ is the ground state spatial wave function of the $i$ th spin component in the $\nu$ th well $(\nu=\mathrm{L}, \mathrm{R})$. Validity of the mean field approximation is discussed in the conclusion part of the paper. As has been studied in great detail $[25,26]$, both spin- 1 condensates of ${ }^{87} \mathrm{Rb}$ and ${ }^{23} \mathrm{Na}$ atoms are dominated by spin symmetric interaction. This leads to the wide use of single spatial mode approximation (SMA) [26], whereby the mode function is taken to be the same of all three components, and is itself determined only from the symmetric interactions. We will adopt this approximation as recent studies have fully delineated its validity regime [26-28]. Denote atomic density as $n_{\nu}(\vec{r})$, hence we take $\phi_{\nu i}$ $=\sqrt{n_{\nu}(\vec{r})}$, which leads to

$$
\Psi(\vec{r}, t)=\sqrt{n_{L}(\vec{r})} \vec{\xi}(t)+\sqrt{n_{R}(\vec{r})} \vec{\eta}(t),
$$

where $\vec{f}_{\nu}\left(\vec{f}_{L}=\vec{\xi}\right.$ and $\left.\vec{f}_{R}=\vec{\eta}\right)$ is the spin-1 spinor associated with the condensate in the $\nu$ th well, and should be interpreted as a column vector, while $\vec{f}_{\nu}^{\dagger}$ a row vector in our notation.

We follow the convention of Ref. [26] to split the total Hamiltonian for an interacting spin-1 condensate into two parts $H=H_{S}+H_{A}$. After integrating over the spatial variables and adopting the SMA introduced above we get 


$$
\begin{gathered}
H_{S}=\sum_{\nu=L, R}\left(\epsilon_{\nu} \vec{f}_{\nu}^{\dagger} \vec{f}_{\nu}+\frac{1}{2} \lambda_{\nu}^{(S)} \vec{f}_{\nu}^{\dagger} \vec{f}_{\nu} \vec{f}_{\nu}^{\dagger} \vec{f}_{\nu}\right)+J\left(\vec{\xi} \vec{\xi}^{\dagger} \vec{\eta}+\vec{\eta}^{\dagger} \vec{\xi}\right), \\
H_{A}=\frac{1}{2} \sum_{\nu=L, R} \lambda_{\nu}^{(A)} \sum_{j=x, y, z} \vec{f}_{\nu}^{\dagger} F_{j} \vec{f}_{\nu} \vec{f}_{\nu}^{\dagger} F_{j} \vec{f}_{\nu},
\end{gathered}
$$

where $F_{j}$ are spin-1 matrices. We have defined $\lambda_{\nu}^{(S / A)}$ $=c_{S / A} \int d \vec{r} n_{\nu}^{2}(\vec{r})$, the single well ground state energy

$$
\epsilon_{\nu}=\int d \vec{r}\left(\frac{\hbar^{2}}{2 M}\left[\nabla \sqrt{n_{\nu}}\right]^{2}+\sqrt{n_{\nu}} V \sqrt{n_{\nu}}\right),
$$

and a positive tunnelling coefficient

$$
J=\int d \vec{r}\left(\frac{\hbar^{2}}{2 M} \nabla \sqrt{n_{L}} \cdot \nabla \sqrt{n_{R}}+\sqrt{n_{L}} V \sqrt{n_{R}}\right) .
$$

The spin symmetric and asymmetric coefficients are $c_{S}$ $=4 \pi \hbar^{2}\left(a_{0}+2 a_{2}\right) / 3 M$ and $c_{A}=4 \pi \hbar^{2}\left(a_{2}-a_{0}\right) / 3 M$ in terms of the scattering length $a_{0}\left(a_{2}\right)$ in the total spin channel of 0 (2) of the two colliding spin-1 atoms. The tunnelling coupling is the same for all spin components as the potential is assumed spin independent, i.e., $V_{i j}=V \delta_{i j}$ for simplicity. The terms involving small spatial overlaps between the wave functions in each well are neglected [19].

The time dependent Schrödinger equations for the spinors are obtained from $i \hbar d \vec{\xi} / d t=\delta H\left(\vec{\xi}, \vec{\eta}, \vec{\xi}^{\dagger}, \vec{\eta}^{\dagger}\right) / \delta \vec{\xi}^{\dagger}$, which gives in a compact form,

$$
\begin{gathered}
i \hbar \frac{d \vec{\xi}}{d t}=\left(\epsilon_{L}+\left(\lambda_{L}^{(S)}+\lambda_{L}^{(A)}\right)|\vec{\xi}|^{2}-\lambda_{L}^{(A)} h_{L}\right) \vec{\xi}+J \vec{\eta}, \\
i \hbar \frac{d \vec{\eta}}{d t}=J \vec{\xi}+\left(\epsilon_{R}+\left(\lambda_{R}^{(S)}+\lambda_{R}^{(A)}\right)|\vec{\eta}|^{2}-\lambda_{R}^{(A)} h_{R}\right) \vec{\eta},
\end{gathered}
$$

the equivalent coupled Gross-Pitaevskii equation for the two spinors. We have defined a shorthand notation $h_{\nu} \equiv{\overrightarrow{f^{\prime}}}_{\nu}^{*} \otimes \vec{f}^{\prime}{ }_{\nu}$ with $\vec{f}^{\prime T}=\left(f_{-},-f_{0}, f_{+}\right)$. Both $\vec{\xi}$ and $\vec{\eta}$ are normalized to unity, so we attempt to find the stationary states satisfying

$$
i \hbar \frac{d}{d t}\left(\begin{array}{c}
\vec{\xi} \\
\vec{\eta}
\end{array}\right)=\mu\left(\begin{array}{l}
\vec{\xi} \\
\vec{\eta}
\end{array}\right)
$$

In this short article, we will focus on the tunnelling dynamics in a symmetric double well with $\epsilon_{L}=\epsilon_{R}=\epsilon, \lambda_{L}^{(S)}$ $=\lambda_{R}^{(S)}=\lambda_{S}, \lambda_{L}^{(A)}=\lambda_{R}^{(A)}=\lambda_{A}$. In this case, interwell tunnelling couples the same spin components, while intrawell interactions cause spin mixing, coupling components $|+\rangle$ and $|-\rangle$ to $|0\rangle$. We have performed extensive simulations and discovered a variety of interesting solutions: self-trapping for each spinor component, for combinations of different spinor components, as well as general nonlinear Josephson-type complete oscillations. To illustrate these results, we will selectively display some oscillation dynamics that might be useful to guide ongoing experimental efforts. For these solutions to be physically meaningful, we have also studied their stabilities by a standard linearization analysis, and only stable solutions are considered.

We now try to formulate the evolution of the most easily

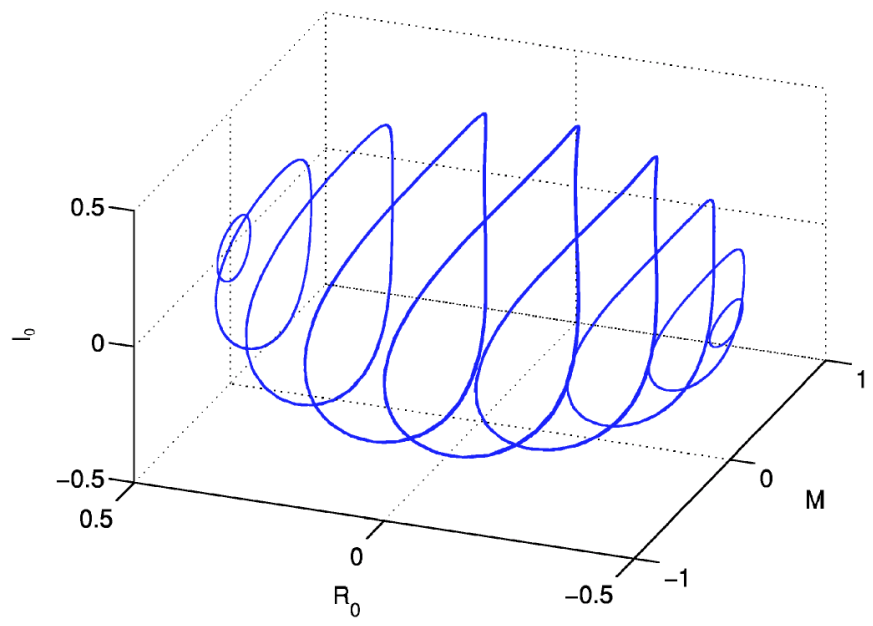

FIG. 1. (Color online) The Josephson phase diagram for $J$ $=0.02$ and $\lambda_{A}=-0.01$. The trajectories of Eq. (9) stay on closed paths. $\lambda_{A}<0(>0)$ refers to a ferromagnetic (antiferromagnetic) spin- 1 condensate as for ${ }^{87} \mathrm{Rb}\left({ }^{23} \mathrm{Na}\right)$ atoms and $\left|\lambda_{A}\right| \ll\left|\lambda_{S}\right|$.

measurable quantity: magnetization in each of the symmetric double wells. Since the total magnetization of our system is a conserved quantity, we therefore only need to consider the magnetization in one of the wells, e.g. the left well. We further consider a special initial state $\eta_{+}=\xi_{-}, \eta_{0}=\xi_{0}$, and $\eta_{-}$ $=\xi_{+}$. The density matrix of the system then becomes $\rho=\vec{\xi}^{*}$ $\otimes \vec{\xi}^{T}$ with $\rho_{i j}=\xi_{i}^{*} \xi_{j}$ for $i, j=+, 0,-$. It may be noted that $\rho$ stands for single-particle density matrix, as the many-body density matrix is beyond the scope of a mean field analysis. The dynamical variables contributing to the evolution of magnetization $M$ are given by

$$
\begin{gathered}
M=\rho_{++}-\rho_{--} ; \quad n_{0}=\rho_{00} ; \\
R_{ \pm}=\frac{1}{2}\left(\rho_{+0} \pm \rho_{0-}+c . c\right) ; \quad R_{0}=\frac{1}{2}\left(\rho_{+-}+\rho_{-+}\right) \\
I_{ \pm}=\frac{1}{2 i}\left(\rho_{+0} \pm \rho_{0-}-c . c\right) ; \quad I_{0}=\frac{1}{2 i}\left(\rho_{+-}-\rho_{-+}\right) .
\end{gathered}
$$

We note that $R_{0}^{2}+I_{0}^{2}=\left[\left(1-n_{0}\right)^{2}+M\right]\left[\left(1-n_{0}\right)^{2}-M\right] / 4$, which comes directly from the definitions of $R_{0}, I_{0}, M$, and the normalization condition for $\vec{f}$. Quite generally, there is a conserved quantity, $R_{+}$, in this system. Further restricting the initial state to $\xi_{0}=0$ we find a simple situation analogous to the standard Josephson junction for a scalar condensate in a double well $[19,29]$

$$
\begin{gathered}
\dot{M}=4 J I_{0}, \\
\dot{R}_{0}=-2 \lambda_{A} I_{0} M, \\
\dot{I}_{0}=2 \lambda_{A} R_{0} M-J M .
\end{gathered}
$$

This set of equations can be interpreted as the familiar two state optical Bloch equations, albeit nonlinear ones, with $R_{0}$ and $I_{0}$ being the analogous real and imaginary parts of the atomic dipole moment. $M$ then acts as the population inver- 


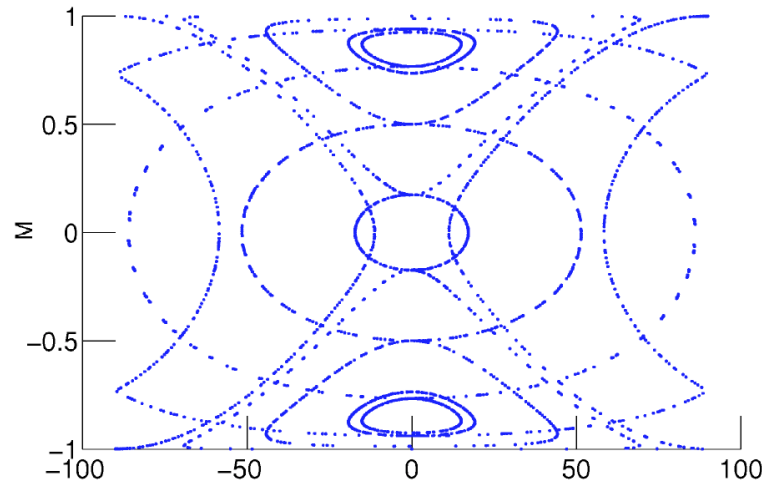

FIG. 2. The phase portrait for $J=0.0051$ and $\lambda_{A}=-0.01$. The angle is in degrees.

sion. The fixed point $I_{0}=M=0$ is a center, which is not an attractor. When $J \leqslant\left|\lambda_{A}\right|$, there exists another fixed point $I_{0}$ $=2 \lambda_{A} R_{0}-J=0$, which is unstable since one of its three characteristic frequencies is positive. Since $M$ is physically bounded between 1 and -1 , we anticipate there exists a limit cycle in this system. For $\xi_{+}=1$, we find $R_{0}=\left(1-M^{2}\right) \lambda_{A} / 4 J$. The phase diagrams for the case of full Josephson oscillations are shown in Fig. 1. Alternatively, we can construct two-dimensional phase portraits by introducing an auxiliary variable $\theta_{+-}=\tan ^{-1}\left(I_{0} / R_{0}\right)$ as illustrated in Fig. 2.

Equation (9) can be analytically solved in terms of the Jacobi elliptic functions [29] with the oscillation period given by

$$
\tau= \begin{cases}2 K\left(\frac{2 J}{\left|\lambda_{A}\right|}\right) /\left|\lambda_{A}\right|, & 2 J<\left|\lambda_{A}\right|, \\ 4 K\left(\frac{\left|\lambda_{A}\right|}{2 J}\right) /(2 J), & 2 J>\left|\lambda_{A}\right|,\end{cases}
$$

where $K($.$) is the complete elliptic integral of the first kind.$ The behavior of the oscillation period with respect to $2 J /\left|\lambda_{A}\right|$ is shown in Fig. 3. As we can see, for small values of $2 J /\left|\lambda_{A}\right|$ the gradually increasing period resembles that of a nonlinear pendulum oscillation, which exhibits self-trapping. The selftrapping condition is therefore $2 J<\left|\lambda_{A}\right| .2 J /\left|\lambda_{A}\right|=1$ is the critical value corresponding to the homoclinic orbit of the equivalent pendulum being completely in the top position. Beyond that the period decreases with increasing $2 J /\left|\lambda_{A}\right|$, as the equivalent pendulum assumes a librator rotation, i.e., a full oscillation of the magnetization. Figure 4 clearly illustrates these different behaviors for the initial state $\vec{\xi}(0)$ $=(1,0,0)$ and $\vec{\eta}(0)=(0,0,1)$.

When the population in the Zeeman state $\left|M_{F}=0\right\rangle$ becomes nonzero, even very small, for instance with $\xi_{+}=\eta_{-}$ $=0.9962, \xi_{0}=\eta_{0}=0.0872$, and $\xi_{-}=\eta_{+}=0$, interaction caused self-trapping disappears, while the full magnetization oscillation reappears in the previously self-trapped regime. Yet, surprisingly, the presence of the nonlinearity due to atomic self-interaction remains visible through dynamic phenomena analogous to self-trapping in the collective variables of other combinations of the single particle density matrix elements. In particular if we examine the collective variable $\rho_{++}-\rho_{00}$,

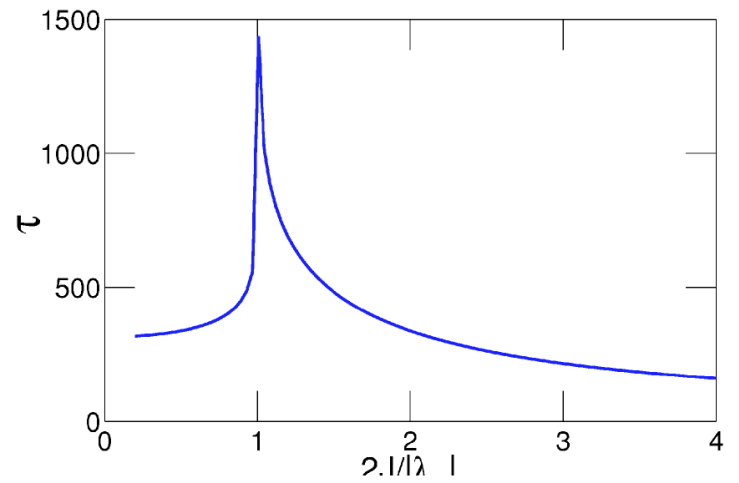

FIG. 3. Dependence of the period to the $2 J /\left|\lambda_{A}\right|$. Period is in seconds.

we find that it does not always exhibit full oscillations. As an example, we demonstrate this effect, unique to a spinor condensate, in Fig. 5 for $\rho_{++}-\rho_{00}$. With the given initial conditions full (complete) oscillations between 1 and 0 occur when the spin dependent atomic self-interaction is turned off, as shown by the dashed line. Inclusion of the self-interaction, on the other hand, inhibits the full oscillation, leading to a similar self-trapping effect as in the nonlinear Josephson dynamics of a scalar condensate in a double well. As a general rule, we find oscillations now become incomplete over any regular interval. The values of $\rho_{++}-\rho_{00}$ can even become negative as a result of including the self-interaction. We can summarize the above observations based on the phase space nonlinear dynamics. When $\lambda_{A}$ is nonzero and in the selftrapping regime when $2 J<\left|\lambda_{A}\right|$, our model system possesses one attractor, which attracts trajectories of different collective variables depending on the initial conditions.

In the opposite regime to self-trapping when $2 J>\left|\lambda_{A}\right|$, the system possesses two distinct attractors, leading to a complete oscillation of magnetization between -1 and 1 . We can adjust the system parameters to shrink the distance between the two attractors in the phase space, and generate beatings in the magnetization oscillation with appropriately chosen initial conditions. The mathematical reason is simple. Due to the presence of the self-interaction in the limit of $2 J>\left|\lambda_{A}\right|$, the orbits of magnetization in the phase space now become open and continuously circulate around two closely spaced focuses (of the two attractors), leading to beating patterns.

Finally, we provide some supporting arguments for the mean field treatment of the tunnelling dynamics. The validity of the mean field theory can be intuitively expected due to the large numbers of atom in our model. At $N \sim 10^{7}$ atoms, our system is beyond the microscopic regime, and becomes at least mesoscopic if not completely macroscopic. We note that the period of magnetization oscillation, given in Eq. (10), has a simple dependence on the number of atoms $\tau$ $\sim 1 / N$. Following the same analysis as in Ref. [30], we can estimate the effect of atom number fluctuations by $|[\tau(N \pm \sqrt{N})-\tau(N)] / \tau(N)| \sim 1 / \sqrt{N}$, which is less than $0.003 \%$ for the present system. We have simply taken the standard deviation $\sigma(N)$ of the atom number fluctuation to be $\sim \sqrt{N}$ consistent with the mean field theory. We can also estimate the absolute phase diffusion times of our system due to atom number fluctuations [31]. The spin symmetric interaction in a 


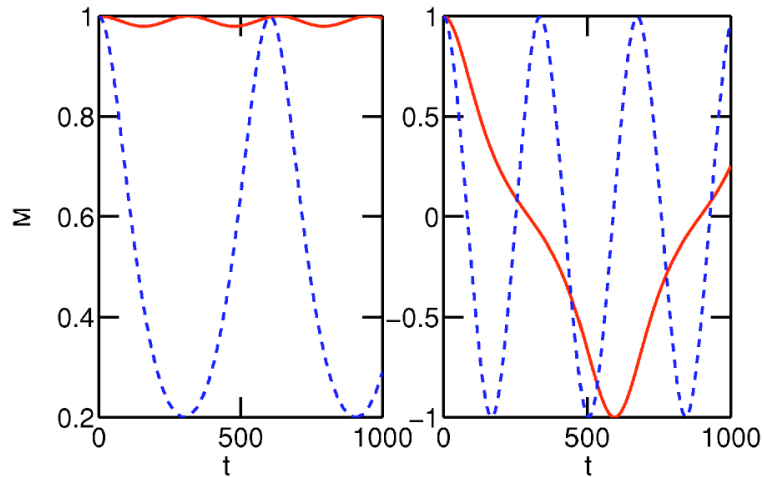

FIG. 4. Initial state is $\vec{\xi}(0)=(1,0,0)$ and $\vec{\eta}(0)=(0,0,1) . \epsilon=1.0$, $\lambda_{S}=1.0$, and $\lambda_{A}=-0.01$. Left figure shows self-trapping when $2 J$ $\leqslant\left|\lambda_{A}\right|$, solid line for $J=0.001$, dashed line for $J=0.0049$. Right figure shows magnetization oscillation, solid line for $J=0.0051$, dashed line for $J=0.01$. Time is in seconds.

spin-1 condensate gives rise to a diffusion time of $\tau_{c}^{(S)}$ $\sim 20 \mathrm{~s}$ for the overall condensate phase, while the spin asymmetric interaction gives rise to a diffusion time of $\tau_{c}^{(A)}$ $\sim 5000 \mathrm{~s}$ for the relative phases between different condensate components. In the above estimates, we have simply taken $\tau_{c}^{(S)} \approx N /\left[\sigma(N) c_{S}\langle n\rangle\right]$ as for a scalar condensate [18] and $\tau_{c}^{(A)} \approx N /\left[\sigma(N) c_{A}\langle n\rangle\right]$ for the spin mixing dynamics [26]. We have also assumed an average condensate density of $\langle n\rangle=1.7 \times 10^{13} \mathrm{~cm}^{-3}$ for $N=10^{7}{ }^{87} \mathrm{Rb}$ atoms, which corresponds to a typical Josephson oscillation period of $\sim 1 \mathrm{~s}$. Thus we conclude that macroscopic magnetization tunnelling in a spinor condensate as modelled here can be examined faithfully with the mean field theory. We further note that in a recent experiment with a smaller number of atoms $N$ $\sim 1000$, the nonlinear Josephson oscillations observed were in complete agreement with the predictions of the mean field theory [32].

In conclusion, we have studied the double well system of a spin-1 atomic condensate using the mean field theory and SMA. We have characterized the various regimes of the nonlinear dynamics for the resulting macroscopic quantum tunnelling phenomena. In addition to features commonly attached to the scalar Josephson dynamics of a single component condensate in a double well, e.g. coherent atomic population oscillation and macroscopic self-trapping [19,29],

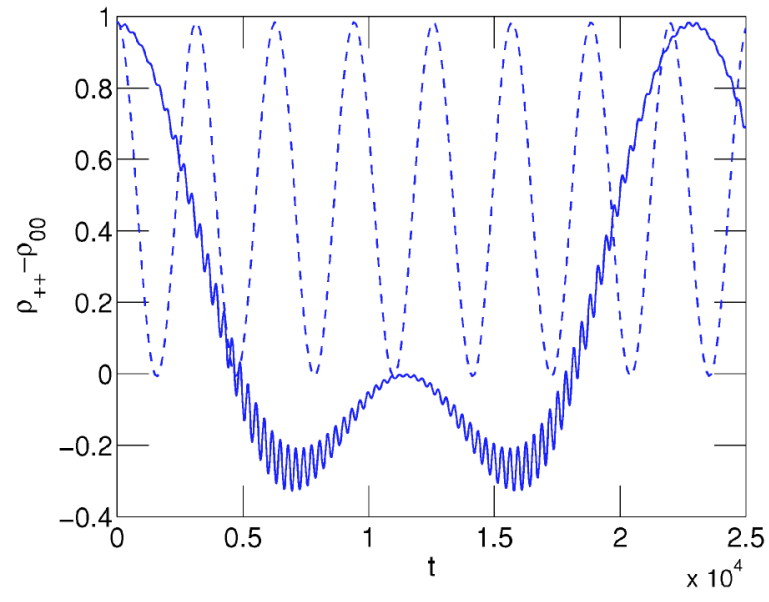

FIG. 5. (Color online) Oscillation of the collective variable $\rho_{++}-\rho_{00}$ for an initial state $\vec{\xi}(0)=(0.9962,0.0872,0)$. Other parameters used are $\epsilon=1.0, \lambda_{S}=1.0, \lambda_{A}=-0.01$ (solid line), and $\lambda_{A}=0$ (dashed line for the case of no spin dependent atomic interaction), and $J=0.001$. Time is in seconds.

we have found interesting effects solely due to the spinor nature of the condensate such as the macroscopic oscillation and self-trapping of condensate magnetization without net changes of total atom numbers within each well. The physics of these correlated tunnelling dynamics is essentially the same as what was found before in a double well system of a two component (or spin 1/2) condensate [23], but can become significantly richer due to the additional freedom in atomic internal states. For instance, amplitude modulations in the magnetization oscillation become possible in a spin-1 condensate. We have illustrated the tunnelling induced macroscopic magnetization oscillations with several examples for a spin-1 condensate of ferromagnetic interactions. Our results highlight the coherent Josephson dynamics of a spinor condensate in a double well, and lay the ground work for future studies of the quantum state of atoms in such a system.

We thank Dr. A. Smerzi for helpful comments and discussions. O.E.M. acknowledges support from a TÜBA/GEBİP award. The work of L. Y. is supported by the NSF and NASA.
[1] A. J. Leggett, S. Chakravarty, A. T. Dorsey, M. P. A. Fisher, A. Garg, and W. Zwerger, Rev. Mod. Phys. 59, 1 (1987); A. J. Leggett, in Chance and Matter, edited by J. Souletie, J. Vannimenus, and R. Stora (North-Holland, Amsterdam, 1987).

[2] A. J. Leggett and A. Garg, Phys. Rev. Lett. 54, 857 (1985).

[3] A. Garg, Europhys. Lett. 22, 205 (1993).

[4] E. M. Chudnovsky and J. Tejada, Macroscopic Quantum Tunneling of the Magnetic Moment, Cambridge Studies in Magnetism (Cambridge University Press, Cambridge, 1998), Vol. 4.

[5] Quantum Tunneling of Magnetization-QTM'94, Vol. 301 of
NATO Advanced Study Institute, edited by L. Gunther and B. Barbara (Kluwer, Dordrecht, 1995).

[6] J. Villain, F. Hartman-Boutron, R. Sessoli, and A. Rettori, Europhys. Lett. 27, 159 (1994).

[7] T. Lis, Acta Crystallogr., Sect. B: Struct. Crystallogr. Cryst. Chem. 36, 2042 (1980).

[8] K. Wieghart, K. Pohl, I. Jibril, and G. Huttner, Angew. Chem., Int. Ed. Engl. 23, 77 (1984).

[9] D. D. Awschalom, J. F. Smyth, G. Grinstein, D. P. DiVincenzo, and D. Loss, Phys. Rev. Lett. 68, 3092 (1992); A. Garg, ibid. 
70, 1541 (1993).

[10] D. M. Stamper-Kurn, M. R. Andrews, A. P. Chikkatur, S. Inouye, H. J. Miesner, J. Stenger, and W. Ketterle, Phys. Rev. Lett. 80, 2027 (1998).

[11] M. Barrett, J. Sauer, and M. S. Chapman, Phys. Rev. Lett. 87, 010404 (2001)

[12] H. Pu, W. Zhang, and P. Meystre, Phys. Rev. Lett. 89, 090401 (2002).

[13] A. Marchenkov, R. W. Simmonds, S. Backhaus, A. Loshak, J. C. Davis, and R. E. Packard, Phys. Rev. Lett. 83, 3860 (1999); S. Backhaus, S. Pereverzev, R. W. Simmonds, A. Loshak, J. C. Davis, and R. E. Packard, Nature (London) 392, 687 (1998).

[14] Q. Niu, X.-G. Zhao, G. A. Georgakis, and M. G. Raizen, Phys. Rev. Lett. 76, 4504 (1996); S. R. Wilkinson, C. F. Bharucha, K. W. Madison, Q. Niu, and M. G. Raizen, ibid. 76, 4512 (1996).

[15] M. Ben Dahan, E. Peik, J. Reichel, Y. Castin, and C. Salomon, Phys. Rev. Lett. 76, 4508 (1996).

[16] D. L. Haycock, P. M. Alsing, I. H. Deutsch, J. Grondalski, and P. S. Jessen, Phys. Rev. Lett. 85, 3365 (2000).

[17] C. Orzel, A. K. Tuchman, M. L. Fenselau, M. Yasuda, and M. A. Kasevich, Science 291, 2386 (2001).

[18] A. Imamoglu, M. Lewenstein, and L. You, Phys. Rev. Lett. 78, 2511 (1997).

[19] G. J. Milburn, J. Corney, E. M. Wright, and D. F. Walls, Phys. Rev. A 55, 4318 (1997).
[20] R. W. Spekkens and J. E. Sipe, Phys. Rev. A 59, 3868 (1999).

[21] T.-L. Ho and C. V. Ciobanu, J. Low Temp. Phys. 135, 257 (2004).

[22] S. Ashhab and C. Lobo, Phys. Rev. A 66, 013609 (2002).

[23] H. T. Ng, C. K. Law, and P. T. Leung, Phys. Rev. A 68, 013604 (2003).

[24] A. J. Leggett, Rev. Mod. Phys. 73, 307 (2001).

[25] T.-L. Ho, Phys. Rev. Lett. 81, 742 (1998); T.-L. Ho and S. K. Yip, ibid. 84, 4031 (2000).

[26] C. K. Law, H. Pu, and N. P. Bigelow, Phys. Rev. Lett. 81, 5257 (1998).

[27] H. Pu, C. K. Law, and N. P. Bigelow, Physica B 280, 27 (2000).

[28] S. Yi, O. E. Müstecaplioğlu, C. P. Sun, and L. You, Phys. Rev. A 66, 011601(R) (2002).

[29] A. Smerzi, S. Fantoni, S. Giovanazzi, and S. R. Shenoy, Phys. Rev. Lett. 79, 4950 (1997); S. Raghavan, A. Smerzi, S. Fantoni, and S. R. Shenoy, Phys. Rev. A 59, 620 (1999); S. Giovanazzi, A. Smerzi, and S. Fantoni, Phys. Rev. Lett. 84, 4521 (2000).

[30] W. Zhang, D. L. Zhou, M.-S. Chang, M. S. Chapman, and L. You Phys. Rev. A (to be published).

[31] S. Yi, Ö. E. Müstecaplioğlu, and L. You, Phys. Rev. Lett. 90, 140404 (2003).

[32] M. Albiez, R. Gati, J. Fölling, S. Hunsmann, M. Cristiani, and M. K. Oberthaler, e-print cond-mat/0411757v2. 